\title{
LA TRADUCCIÓN EN LOS MANUALES DE ESPAÑOL PARA EXTRANJEROS EN ALEMANIA
}

\author{
Alicia Escribano Sabio \\ alicia_escribano@yahoo.es \\ Universidad de Heilbronn
}

Resumen: El objetivo de este artículo es estudiar el papel que los manuales de ELE, utilizados en el aula alemana universitaria para los niveles $A 1$ y $A 2$, le atribuyen a la traducción en clase. Para ello, llevaré a cabo un análisis y comparación de las actividades de traducción de manuales actuales, así como de su objetivo en el proceso de enseñanza-aprendizaje. Asimismo, expondré brevemente, la importancia de este recurso en clase.

Palabras clave: traducción, lengua materna, lengua meta, metodología, aprendizaje de una lengua extranjera.

Abstract: The main aim of this paper is to discuss the role of translation in elementary Spanish language learning textbooks (A1-A2) used in the German higher education context. To that end, some translation activities included in current textbooks will be analysed and compared with regard to their teaching and learning objectives. Furthermore, this study will argue the pivotal role of translation as a resource in second language class.

Keywords: translation, native language, target language, methodology, second language learning. 


\section{A. Escribano Sabio}

2019. "La traducción en los manuales de español para extranjeros en Alemania" Sabir. International Bulletin of Applied Linguistics, 1: 52-66

\section{Introducción}

En la historia de la enseñanza de una lengua extranjera, la traducción ha representado un instrumento esencial y frecuente usado por los diferentes métodos y enfoques. Sin embargo, en la actualidad es un tema que genera controversia, ya que algunos autores se posicionan, tanto a favor de que el profesor traduzca en clase, como de que se usen actividades de traducción. Otros, por el contrario, abogan por una metodología que evite el uso de esta técnica en el aula.

En la enseñanza de ELE, la traducción como herramienta única supone una técnica claramente desaconsejable; no tendría sentido limitar la clase a traducir todo el tiempo como se hacía en métodos antiguos. Sin embargo, usar este instrumento como una ayuda para el proceso de enseñanza-aprendizaje de lenguas extranjeras, sí es una opción viable que, en nuestra opinión, presenta ventajas. Hernández se manifiesta al respecto opinando lo siguiente:

“No se trata de traducir, sino de lograr que el aprendiente perfeccione la L2 tomando una mayor conciencia de dicha lengua a través de un texto; la traducción como actividad didáctica nos permite trabajar los conocimientos léxicos, gramaticales y pragmáticos de una manera precisa."

(Hernández, 1996: 253)

Es conveniente aclarar que el tipo de traducción al que hago alusión en este trabajo es la traducción pedagógica: "es la que se usa en el aula con un fin instrumental, cuya finalidad es transmitir el mensaje de los textos originales a un público que desconoce la lengua en que están producidos dichos textos" (Capel Moreno, 2005: 80). 
A la vista de todo ello, el objetivo de este trabajo es estudiar el papel que los manuales comunicativos de editoriales alemanas de ELE le dan a las actividades en las que prima la lengua materna del alumno. Expondré mis creencias, según mi experiencia como profesora en la universidad alemana, sobre qué puntos positivos aportan estas actividades en el desarrollo del aprendizaje.

\section{Metodología}

En el presente trabajo me centraré en el uso de la traducción en el aula alemana universitaria de ELE. Para ello, analizaré los principales manuales actuales de Enfoque Comunicativo utilizados en las aulas alemanas, correspondientes a los niveles A1, A2 y B1, que presentan algunas actividades que hacen uso de esta técnica.

\section{La traducción en la historia de las diferentes metodologías para la enseñanza de lenguas extranjeras}

En referencia a los métodos usados en el pasado destacaremos, concretamente, los que hemos considerado más importantes a lo largo de la historia de la enseñanza de una lengua extranjera: el Método de Gramática-Traducción, el Método Directo y el Enfoque Comunicativo, usado en nuestros días ${ }^{1}$.

El primer método, el de Gramática- traducción, como su propio nombre indica, gira en torno a la enseñanza de la gramática de la lengua meta. Se practica mayormente mediante actividades de traducción directa e inversa, por lo que prima la técnica de traducir en clase. Dominó la enseñanza de lenguas desde la primera mitad

\footnotetext{
${ }^{1}$ Para una revisión panorámica de la enseñanza de lenguas extranjeras, cfr. García Marcos (2018).
} 


\section{A. Escribano Sabio}

2019. "La traducción en los manuales de español para extranjeros en Alemania" Sabir. International Bulletin of Applied Linguistics, 1: 52-66

del siglo XIX hasta la primera mitad del siglo XX. El léxico se aprendía a través de listas de palabras, sin estar contextualizadas y, para conocer el significado de estas, se recurría al diccionario. El profesor sería también la figura más importante en clase, destacando su papel de protagonismo frente al discente, cuyo papel era secundario.

En respuesta a este modo de trabajo en el aula, surgiría el Método Directo, el cual abogaba por la prohibición del uso de la lengua materna en clase y, por lo tanto, el uso de esta para traducir lo que no se entendía. Sánchez nos describe la postura de este método con las siguientes palabras:

"La traducción está terminantemente prohibida: el significado de las palabras y de las frases se adquiere recurriendo a cualquier mecanismo o ayuda, pero nunca mediante la traducción. Se da por supuesto que el recurso de la traducción dificulta la asociación directa significante-significado."

(Sánchez, 2009: 64)

La lengua meta se aprendía recurriendo, o bien a imágenes, o bien a gestos del profesor. Se utilizaba, frecuentemente, la fórmula pregunta-respuesta entre alumnoprofesor. El énfasis se ponía en la lengua oral, primando así la conversación y relegando la expresión escrita a un segundo plano, a diferencia del Método de GramáticaTraducción. El léxico se aprendería siempre en un determinado contexto y no mediante listas de palabras. Se perseguía la práctica oral a través de situaciones comunicativas cotidianas.

Actualmente el método mayormente utilizado es el Enfoque Comunicativo. Las premisas difieren, considerablemente, de los métodos anteriores puesto que en él se pone, principalmente, énfasis en la práctica oral, dando primicia al significado y buscando una comunicación que sea efectiva, estimulando al discente desde el principio a la conversación. Pastor Cesteros lo define de la siguiente manera: 


\section{A. Escribano Sabio}

2019. "La traducción en los manuales de español para extranjeros en Alemania" Sabir. International Bulletin of Applied Linguistics, 1: 52-66

"Lo que sí lo identifica en cualquier caso es el planteamiento de la competencia comunicativa como principal objetivo de la enseñanza de idiomas, mediante el desarrollo de las cuatro destrezas lingüísticas. La visión del aprendizaje basado en la experiencia y centrado en el alumno también define este enfoque."

(Pastor Cesteros, 2004: 158)

En contraposición a los métodos anteriormente mencionados, en este se acepta la traducción en clase: "se puede usar la traducción cuando los alumnos la necesiten o se beneficien de ella". (Richards y Rodgers, 2009: 157).

Littlewood señala las ventajas e inconvenientes del uso de la lengua materna en el proceso de aprendizaje-enseñanza de una lengua extranjera:

"Muchos profesores usan la lengua materna de los estudiantes, decisión que puede ser a menudo necesaria en interés de organizar las clases con claridad y eficacia. Sin embargo, también supone sacrificar valiosas oportunidades para usar la lengua extranjera con una buena motivación. Además, tiende a devaluar la lengua extranjera como vehículo de comunicación: los estudiantes la ven asignada a campos que no son comunicativamente esenciales, como los ejercicios de repetición y transformación o la práctica de diálogos, mientras que la lengua materna sigue siendo el medio apropiado para comentar los temas de importancia."

(Littlewood, 2012: 43)

Podemos concluir, a la vista de todo ello, que la traducción en la enseñanza de lenguas quedó anquilosada tras el Método de Gramática-Traducción y se desterró totalmente del aula, considerándose como algo aburrido, obsoleto y contraproducente, ya que mantenía al alumno en silencio en el aula y sin comunicarse. Sin embargo, como veremos a continuación en las actividades que analizamos en este 


\section{A. Escribano Sabio}

2019. "La traducción en los manuales de español para extranjeros en Alemania" Sabir. International Bulletin of Applied Linguistics, 1: 52-66

estudio, se pueden plantear desde otros puntos de vista, cambiando la manera de trabajar, sin que en clase prime, por supuesto, esta herramienta. Actualmente, se usa como un recurso más en el aula de ELE, el cual ayuda a comparar la lengua materna y la lengua meta.

\section{Análisis: actividades de traducción y mediación en manuales de ELE de editoriales alemanas}

El presente análisis muestra diferentes tipos de actividades que ofrecen distintos manuales, en su mayoría actuales, en el aula universitaria de ELE que utilizan la lengua materna del estudiante. Entre estas, podemos destacar las siguientes:

1. Actividades de mediación. Aparecen en el manual Meta profesional A1-A2, el cual está orientado a la enseñanza de español con Fines Específicos (EFE), concretamente al mundo laboral. Se presentan cada tres lecciones, en un apartado llamado "etapas". En ellas, los alumnos deben interpretar la información que aparece en una determinada actividad presentada en español, la cual transmitirán (de forma oral o escrita) a su compañero en su lengua materna; en este caso, en alemán. Un ejemplo de este tipo de actividades es el siguiente:

- Leer un folleto y extraer una determinada información que los estudiantes tienen que contar a su jefe mediante una carta escrita en alemán.

El manual consta de un total de 5 actividades de mediación, en las cuales, tras leer y escuchar una determinada información en español, informará al compañero lo que ha logrado entender, pero siempre a través de su lengua materna. 


\section{A. Escribano Sabio}

2019. "La traducción en los manuales de español para extranjeros en Alemania" Sabir. International Bulletin of Applied Linguistics, 1: 52-66

2. Actividades de traducción directa de alemán a español. Los manuales analizados, en esta ocasión, han sido Caminos plus A1-A2, Perspectivas iya! $A 1$ y su correspondiente volumen $\mathrm{A} 2 \mathrm{y}$, Con gusto A1, y el siguiente, $\mathrm{A} 2$. Todos ellos ofrecen la traducción directa en el cuaderno de ejercicios del método. Consisten en traducir oraciones presentadas en alemán a español. Presentamos, a continuación, un ejemplo:

- Seit 1999 ist er Spanischlehrer².

3. Actividades que explican determinadas situaciones en alemán, y se les pide a los estudiantes que, o bien reaccionen antes estas situaciones, o bien escriban cómo se expresarían en estos contextos. Un ejemplo de estas es el siguiente:

- Wie begrüßen Sie Ihre Freundin nach dem Mittagessen?³- ¿Cómo saluda usted a una amiga después del almuerzo?

Como vemos, es una actividad en la que no hay que traducir directamente. Sin embargo, se ofrece una serie de directrices en alemán para poder, así, reaccionar en español. En el ejemplo anteriormente expuesto, observamos que, en lugar de escribir simplemente "buenas tardes" se presenta la situación, de tal manera que se evita traducir literalmente. Este tipo de actividades nos las encontramos también en los manuales anteriormente mencionados, Caminos plus A1-A2 y Perspectivas iya! A1 y su nivel A2, Con gusto A1 y Con gusto A2, Con gusto nuevo A1. Se practican, también, para hacer diálogos en grupos de dos. Suelen ser temas que se corresponden con situaciones reales como, por ejemplo, comprar un billete de tren, una llamada telefónica, etc.

4. Actividad en la que también hay que traducir de alemán a español con un determinado objetivo relacionado con el aprendizaje de léxico y la distinción de

\footnotetext{
${ }^{2}$ Traducción: "desde 1999 él es profesor".

${ }^{3}$ Ejemplo extraído del manual de ejercicios del método Perspectivas iya! A1. (2012: 10).
} 


\section{A. Escribano Sabio}

2019. "La traducción en los manuales de español para extranjeros en Alemania" Sabir. International Bulletin of Applied Linguistics, 1: 52-66

diferentes vocablos entre las dos lenguas. Citamos, a continuación, algunos ejemplos:

- Los distintos verbos que existen en español para expresar el verbo "werden" en alemán.

- Presentación de numerosos ejemplos en alemán, cuyo objetivo es que el estudiante traduzca, eligiendo correctamente entre los verbos "quedar" o "quedarse", respectivamente.

- Otro ejemplo significativo sería el de los diferentes verbos que existen en alemán para expresar "gustar". Se ofrecen oraciones escritas en alemán con los diferentes verbos que esta lengua contempla para expresar "gustar" y el estudiante tiene que traducirlas al español. Veamos un ejemplo:

- Wie sagen Sie...

....Ihnen Ihre Arbeit (nicht) gefällt? ... dass Sie Diskotheken nicht mögen $?^{4}$ (Con gusto A1, 2012: 126).

5. Actividad de comunicación y mediación. Estas nos las encontramos en un pequeño cuaderno de ejercicios del método Perspectivas ; Ya! $A 1$, junto con el nivel A2. Se utiliza, en mayor parte, la lengua materna de los alumnos con diferentes fines:

- Para traducir directamente de español a alemán y viceversa.

- Para expresar en la lengua meta cómo reaccionaría ante determinadas situaciones, las cuales se describen en alemán.

- Para escribir un diálogo cuya información aparece expuesta en la lengua del discente. No es una traducción literal, sino que se les describe lo que deben comunicar.

\footnotetext{
${ }^{4}$ Traducción: ¿cómo dice usted..., que le gustan las discotecas? ¿ ¿ ... que le gusta su trabajo?
} 
6. En el manual Perspectivas iya! A1 comentado en el punto anterior se ofrece, además, un tipo de actividad que no hemos encontrado en ninguno de nuestros manuales analizados. Se trata de traducir una oración del español a la lengua alemana de forma literal, cuyo resultado, es una producción errónea, pero permite que los alumnos sean conscientes de que hay muchos aspectos de la lengua que se expresan de forma diferente. El manual describe esta actividad de la siguiente manera: "eine wortwörtliche Übersetzung ins Deutsche hilft uns, die Stellung der Wörter im Spanischen zu verdeutlichen ${ }^{5 "}$. Presentamos un ejemplo de este recurso:

- "Esta tarde he bebido mucho vino tinto", cuya traducción en alemán palabra por palabra, sería: "diesen Abend habe getrunken viel Wein rot. Esta traducción literal, en alemán, es errónea.

7. Otro tipo de actividad es aquella en la que se propone traducir, una vez más, algunas oraciones de alemán a español, pero en esta ocasión, la novedad radica en que el estudiante introduce datos sobre él. Veamos un ejemplo:

- Ich habe vor... Jahren begonnen, Spanisch zu lernen ${ }^{6}$. (Con gusto nuevo A2, 2019: 113).

A la vista de este ejemplo, podemos observar que el estudiante, además de traducir la oración, tiene la oportunidad de escribir un dato personal: "hace... empecé a estudiar español". Se persigue, por tanto, en esta actividad, esclarecer la diferencia entre "hace", "desde", "desde hace" debido a que en alemán, para expresar estas tres preposiciones de tiempo, existen solo dos (seit, vor). "Seit" se corresponde con "desde" y "desde hace". Con esta actividad, se tiene la opción de poder diferenciarlas y practicarlas mediante los ejemplos de traducción literal. Este aspecto de la lengua es más complicado en español que en alemán

\footnotetext{
${ }^{5}$ Una traducción palabra por palabra en alemán, nos ayuda a aclarar la posición (orden) de las palabras en español.

${ }^{6}$ Traducción: "hace ... empecé a estudiar español".
} 


\section{A. Escribano Sabio}

2019. "La traducción en los manuales de español para extranjeros en Alemania" Sabir. International Bulletin of Applied Linguistics, 1: 52-66

$y$, con este tipo de práctica, el discente tiene la oportunidad de poder diferenciar mejor el equivalente a su lengua.

8. El manual Power Grammatik Spanisch introduce actividades en las que combina ambas lenguas, recurriendo frecuentemente a la lengua maternal del alumno. No se utiliza como manual de cabecera pero sí como complemento para las clases y como refuerzo para el alumno en casa. A continuación, podemos observar distintos ejemplos de ello:

- Juana es secretaria y trabaja en (einem Büro) (Power Grammatik Spanisch, 2004: 13).

- El lunes es el (erste Tag) de la semana. (Power Grammatik Spanisch, 2004: 21).

- Toca el piano (besser) que su hermana. (Power Grammatik Spanisch, 2004: 29).

9. Por último, en los manuales Con gusto (niveles A1 y A2) y Con gusto nuevo (niveles A1 y A2) encontramos actividades encaminadas concretamente al aprendizaje del léxico. Consisten en mostrar dos columnas de vocabulario, una en alemán y otra en española, en las que el alumno debe unir las palabras con sus traducciones correspondientes. A continuación, presentamos un ejemplo:

- 1. La oferta

2. El pedido

3. El descuento

4. La factura

5. El importe

(..) a. Bestellung

b. Betrag

c. Ladenpreis

d. Rabatt

e. Angebot

(...)

(Con gusto nuevo A1, 2019: 121).

El objetivo de cada uno de estos ejercicios es poder practicar la gramática vista en la lección, la cual, se da con anterioridad en alemán. Se ofrece este tipo de actividad 


\section{A. Escribano Sabio}

2019. "La traducción en los manuales de español para extranjeros en Alemania" Sabir. International Bulletin of Applied Linguistics, 1: 52-66

en casi todas las lecciones. No es un manual apto para usar en clase a diario, pero sí para explotar algunas de sus actividades, bien brevemente en el aula, o en casa como deberes. Con esto, se puede reforzar la gramática, compararla entre las dos lenguas, y poder luchar, de alguna manera, con las interferencias.

La búsqueda de actividades en las que se utilice la traducción no terminó aquí. Se ha realizado un rastreo en otros manuales muy utilizados en la universidad, de editoriales alemanas y de relativa reciente publicación, pero no hemos encontrado ninguno que ofrezca ejercicios de traducción. Entre ellos podemos citar Universo.Ele, en los niveles, A1 y A2 y Estudiantes.Ele, igualmente en ambos niveles.

\section{Resultados.}

Tras el análisis de las actividades de traducción en determinados manuales de clase, se puede constatar que se usan entre dos-tres ejercicios de traducción por lección, mayormente y como era lógico por otra parte, presentados en el cuaderno de ejercicios que complementa con el libro del alumno. Esto pone de relieve que las editoriales alemanas, en su mayoría, son conscientes del papel que juega la lengua materna del alumno en el aprendizaje. Se dan actividades de varios tipos, como hemos observado anteriormente que se pueden concretar de la siguiente manera:

- Actividades de mediación.

- Actividades de traducción directa escrita de alemán a español.

- Actividades que ofrecen directrices en alemán para que sean interpretadas y escritas en español.

- Traducción de alemán a español, cuyo objetivo es la mejora de algún aspecto gramatical o léxico que es de especial dificultad para el aprendizaje.

- Actividades de comunicación. 


\section{A. Escribano Sabio}

2019. "La traducción en los manuales de español para extranjeros en Alemania" Sabir. International Bulletin of Applied Linguistics, 1: 52-66

- Actividades que traducen de español a alemán de forma literal, lo que causa errores en alemán para, así, hacer conscientes a los estudiantes de que las estructuras de ambas lenguas son muy diferentes.

\section{Conclusiones}

Tras el análisis llevado a cabo podemos extraer las siguientes conclusiones:

En primer lugar, las editoriales de manuales de ELE son conscientes de la importancia de este tipo de ejercicios para el estudiante universitario alemán. Observamos, a su vez, que la mayoría de ellos son presentados en los cuadernos de actividades de gran parte de los manuales con los que actualmente se trabaja.

En segundo lugar, podemos considerar la traducción como una herramienta didáctica muy válida ya que, la lengua materna del estudiante va a ejercer siempre un papel influyente, pese a las discrepancias que encontramos en alguna de la bibliografía, en la adquisición de una lengua extranjera. Mediante la traducción se aprende a perfilar las estructuras gramaticales y compararlas entre las dos lenguas y, además es una ayuda importante en la adquisición del léxico puesto que permite descubrir los falsos amigos y encontrar equivalentes entre la lengua de partida y la lengua meta.

En tercer lugar, observamos que los manuales van aportando continuamente novedades en el tipo de ejercicios que ofrecen. El mercado se renueva frecuentemente y esto se refleja directamente en la metodología de trabajo de los manuales. En ese sentido son destacables las actividades de mediación, las de traducción literal con huecos para introducir, de esa manera, información personal del alumno y las que 


\section{A. Escribano Sabio}

2019. "La traducción en los manuales de español para extranjeros en Alemania" Sabir. International Bulletin of Applied Linguistics, 1: 52-66

ofrecen una serie de directrices en la lengua materna para poder, así, reaccionar en la lengua meta.

En cuarto lugar, se trata de ayudar en el proceso de aprendizaje de la lengua mediante traducciones que, en la mayoría de los casos, se remiten a situaciones cotidianas diarias y no a la mera traducción de textos literarios como se hacía en métodos antiguos.

Por último, no hay que olvidar que, puesto que se trata de alumnos que no están en situación de inmersión, estos le dan gran importancia a su lengua materna ya que les sirve de apoyo en su proceso de aprendizaje. Además, proponerlas para, posteriormente trabajarlas en casa es un complemento perfecto para afianzar lo que han aprendido y practicado en clase. También es interesante el trabajo en grupos, para que los alumnos puedan intercambiar opiniones, y teniendo en cuenta las limitaciones de tiempo que se dan en una clase. Intentamos, de este modo, tener más tiempo para las actividades comunicativas y la práctica de todas las destrezas lingüísticas sin que prime, obviamente, la traducción. Recalcamos, con esto, el uso de la traducción como herramienta de apoyo didáctico.

Este es un campo de investigación en el que aún no se han realizado muchos estudios prácticos que nos permitan comprobar el papel de la traducción en clase ELE y las ventajas-desventajas del tipo de actividades analizado en el presente estudio. Habrá que seguir explorando en este campo para que futuras investigaciones nos revelen resultados que nos aporten más datos sobre la eficacia de este recurso en el aula de lenguas extranjeras. 


\section{A. Escribano Sabio}

2019. "La traducción en los manuales de español para extranjeros en Alemania" Sabir. International Bulletin of Applied Linguistics, 1: 52-66

\section{Bibliografía}

\section{Referencias}

Capel Moreno, A, I.

2005. El diseño de tareas de Traducción en Interpretación: un instru-mento en la enseñanza-aprendizaje del inglés como lengua extranjera en bachillerato. Almería: Universidad de Almería.

García Marcos, F.

2018. La trastienda de la enseñanza de lenguas extranjeras. Granada: Comares.

Hernández, M. R.

1996. "La traducción pedagógica en la clase de ELE" en Actas del VII Congreso Internacional de ASELE. Disponible en: https://cvc.cervantes.es/ensenanza/biblioteca_ele/asele/pdf/07/07_0247.pdf (Consultado el día 17 de octubre de 2019)

Littlewood, W.

2012. La enseñanza comunicativa de idiomas. Introducción al Enfoque Comunicativo. Segunda reimpresión. Madrid: Edinumen.

\section{Pastor Cesteros, S.}

2004. Aprendizaje de segundas lenguas. Lingüística aplicada a la enseñanza de idiomas. Alicante: Universidad de Alicante.

Richards, J., C. y T. S. Rodgers.

2009. Enfoques y métodos en la enseñanza de idiomas. Madrid, Edinumen.

Rudolph, H., 2004. Power Grammatik Spanisch. Ismaning: Hueber. 


\section{A. Escribano Sabio}

2019. "La traducción en los manuales de español para extranjeros en Alemania" Sabir. International Bulletin of Applied Linguistics, 1: 52-66

Sánchez Pérez, A.

2009. La enseñanza de idiomas en los últimos cien años. Métodos y enfoques. Madrid: Sgel.

Selinker, L y M. S: Gass

1994. Second language acquisition. An Introductory Course. Segunda edición. Nueva York y Londres: Taylor \& Francis.

\section{Manuales de ELE}

VV. AA.

2009. Con gusto A1. Stuttgart: Klett.

2010. Con gusto A2. Stuttgart: Klett.

2012. Perspectivas ;Ya! A1. Berlín: Cornelsen.

2014. Meta profesional A1-A2. Stuttgart: Klett.

2014. Perspectivas ;Ya! A2. Berlín: Cornelsen.

2017. Estudiantes. ELE A1. Stuttgart: Klett.

2018. Con gusto nuevo A1. Stuttgart: Klett.

2018. Estudiantes. ELE A2. Stuttgart: Klett.

2019. Con gusto nuevo A2. Stuttgart: Klett. 